\title{
Treatment of esophageal anastomotic leakage with self-expanding metal stents: analysis of risk factors for treatment failure
}

Authors

Institutions
Saga Persson ${ }^{1}$, loannis Rouvelas ${ }^{1}$, Koshi Kumagai ${ }^{1}$, Huan Song ${ }^{2}$, Mats Lindblad ${ }^{1}$, Lars Lundell ${ }^{1}$, Magnus Nilsson ${ }^{1}$, Jon A. Tsai ${ }^{1}$

${ }^{1}$ Division of Surgery, Department of Clinical Science Intervention and Technology, Karolinska Institutet, and Centre for Digestive Diseases, Karolinska University Hospital, Stockholm, Sweden

${ }^{2}$ Department of Medical Epidemiology and Biostatistics, Karolinska Institutet, Stockholm, Sweden submitted

22. September 2015

accepted after revision

29. January 2016

\section{Bibliography}

Dol http://dx.doi.org/

10.1055/s-0042-102878

Published online: 30.3.2016

Endoscopy International Open

2016; 04: E420-E426

(c) Georg Thieme Verlag KG

Stuttgart · New York

E-ISSN 2196-9736

\section{Corresponding author}

\section{Jon A. Tsai, MD}

Division of Surgery, K53

Department of Clinical Science Intervention and Technology Karolinska Institutet Centre for Digestive Diseases Karolinska University Hospital 14186 Stockholm Sweden

Fax: +49-8-585-823-40 jon.tsai@ki.se

\section{License terms}

(ब)(1) $\ominus \circledast$
Background and study aim: The endoscopic placement of self-expandable metallic esophageal stents (SEMS) has become the preferred primary treatment for esophageal anastomotic leakage in many institutions. The aim of this study was to investigate possible risk factors for failure of SEMSbased therapy in patients with esophageal anastomotic leakage.

Patients and methods: Beginning in 2003, all patients with an esophageal leak were initially approached and assessed for temporary closure with a SEMS. Until 2014, all patients at the Karolinska University Hospital with a leak from an esophagogastric or esophagojejunal anastomosis were identified. Data regarding the characteristics of the patients and leaks and the treatment outcomes were compiled. Failure of the SEMS treatment strategy was defined as death due to the leak or a major change in management strategy. The risk factors for treatment failure were analyzed with simple and multivariable logistic regression statistics.

\section{Introduction \\ $\nabla$}

Leakage from an esophagogastric or esophagojejunal anastomosis after esophagectomy or gastrectomy is associated with a high postoperative mortality rate and an impaired quality of life during long-term follow-up [1 -6]. Leakage rates vary between $3 \%$ and $25 \%$ after esophagectomy [7-10] and between $3 \%$ and $11 \%$ after total gastrectomy $[3,5,11,12]$. There is a lack of consensus regarding the management of leakage from an esophageal anastomosis because of the rarity of this complication, substantial variability in the severity of the clinical manifestations of the anastomotic dehiscence, and above all the existing literature, which mainly consists of small, single-institution series containing a multiplicity of confounders. As a result, there is only weak evidence in favor of any of the alternative therapeutic options.
Results: A total of 447 patients with an esophagogastric or esophagojejunal anastomosis were identified. Of these patients, $80(18 \%)$ had an anastomotic leak, of whom 46 (58\%) received a stent as first-line treatment. In 29 of these 46 patients, the leak healed without any major change in treatment strategy. Continuous leakage after the application of a stent, decreased physical performance preoperatively, and concomitant esophagotracheal fistula were identified as independent risk factors for failure with multivariable logistic regression analysis.

Conclusion: Stent treatment for esophageal anastomotic leakage is successful in the majority of cases. Continuous leakage after initial stent insertion, decreased physical performance preoperatively, and the development of an esophagotracheal fistula decrease the probability of successful treatment.

Owing to the high rates of mortality and morbidity following traditional open surgical interventions for anastomotic leakage $[9,13]$, several minimally invasive endoscopic methods, which include esophageal stents, clips, fibrin glue, and endoluminal vacuum therapy, have been advocated and introduced [14-16]. Experiences of stent therapy, which is an established therapeutic option for various other causes of gastrointestinal leakage, including spontaneous or iatrogenic perforation, have been described in a few series containing sufficient numbers of patients $[17,18]$; success rates ranging from $54 \%$ to $77 \%$ have been reported [14,19-21]. Most publications have, however, reported experiences based on a small number of cases in which the focus has been on the clinical characteristics of the anastomotic leaks and the outcomes, without further analysis of the factors associated with positive or negative 
outcomes. The significant variations in reported success rates after stent treatment, in addition to the diverse clinical presentations and therapeutic outcomes, emphasize the need for a complementary analysis of the role of stent therapy in the management of esophageal anastomotic leakage. Herein, we report a single-center experience of stent treatment for esophagogastric and esophagojejunal anastomotic leakage that ranges over more than a decade, with a focus on assessing the risk for failure of the SEMS-based therapeutic concept.

\section{Material and methods}

$\nabla$

\section{Patient inclusion}

Beginning in 2003, all patients with a leak from the esophagus were initially approached and evaluated for temporary closure with a self-expandable metallic esophageal stent (SEMS). Until 2014, all patients at the Karolinska University Hospital with a leak from an esophagogastric or esophagojejunal anastomosis were identified. Data regarding the characteristics of the patients and leaks and the treatment outcomes were compiled. All included patients had undergone surgery for malignant or benign disease with esophagectomy and esophagogastric anastomosis, gastrectomy and esophagojejunal anastomosis, or esophagogastrectomy and esophagojejunal anastomosis. Gastrectomy also included proximal gastrectomy/distal esophagectomy with jejunal interposition (Merendino procedure). Patients who had gastrojejunal or esophagocolonic leaks were not included in this study. Patients were identified through the hospital databases Vis-Portalen, TakeCare, and Orbit.

\section{Definition of anastomotic leakage and management}

In case an anastomotic leak was clinically suspected during the postoperative period, the patient underwent either acute endoscopy or, more often, endoscopy preceded by computed tomography with native-phase images and then imaging after the oral intake of water-soluble contrast medium to document and characterize the dehiscence. Anastomotic leakage was defined by the presence of extraluminal collections of air or contrast, excessive amounts of bile-stained fluid, or a combination of these. A diagnosis of leakage could also be established if blue-stained output from a drain or a cervical incision was observed after the patient had orally ingested methylene blue.

If further evaluation of the leakage or any type of intervention was indicated, the patient underwent endoscopy under general anesthesia. During the study period, sealing of the perforation with an esophageal stent was the preferred primary strategy whenever feasible. A fully or partially covered SEMS was inserted over a guidewire under fluoroscopic guidance. Over the 10 -year period, different types of stents were used. When needed, double stents were deployed for better coverage of the defect. After a stent had been inserted, computed tomography was again performed in most cases to exclude persistent leakage. All patients received broad-spectrum antibiotics and often also antifungal drugs.

A tailored approach for optimizing drainage was applied; methods used included percutaneous drainage, drainage via the cervical incision, drainage of the pleural spaces and mediastinum via thoracotomy or thoracoscopy, and trans-hiatal drainage via laparotomy. Patients received parenteral or enteral nutrition until the leakage was considered to be under control. Extraction of the stent, repeated endoscopic inspection of the leak, and the appli- cation of a new stent were regularly performed based on clinical developments and the patient's recovery. Stents were not used in patients with minor subclinical leakage that was judged to be manageable with drainage alone, typically via the cervical incision in those with a proximal neck anastomosis. The SEMS-based concept was not followed in the most severe clinical situations; if signs of conduit necrosis were observed, immediate surgical intervention and conduit takedown were required.

\section{Definition of treatment failure}

Failure was defined as a need for reoperation because of uncontrolled sepsis and mediastinitis (usually rescue esophagectomy with end-esophagostomy) or as death resulting from leakage or the development of an esophagotracheal fistula that did not heal after repeated attempts at management with esophageal stents, usually combined with tracheal stents, and therefore required surgical reconstruction at a later stage [22]. Persistent leakage after stenting in which the leakage and infection could be managed successfully with drainage procedures and antibiotics and eventually healed was accordingly not considered a failure.

\section{Possible risk factors}

The following variables were analyzed as possible risk factors for unsuccessful or failed stent therapy: age; sex; American Society of Anesthesiologists (ASA) physical status classification; smoking; alcohol abuse; cardiovascular disease, pulmonary disease, or diabetes; maximal physical performance on a preoperative bicycle test; indication for surgery (malignant vs. benign disease); prior neoadjuvant treatment; type of resection; type of reconstruction; level of anastomosis; time from surgery to the diagnosis of leakage and stenting; sealed leakage after index stent insertion; number of endoscopic reinterventions (to control leakage); development of esophagotracheal fistula; body mass index (BMI) and C-reactive protein (CRP) level; albumin and creatinine levels preceding stent insertion.

\section{Secondary outcomes and descriptive data}

These included the following: frequency of treatments other than SEMS placement as up-front therapy, days in intensive care unit (ICU), days in hospital, need for ventilator support or hemodialysis, and in-hospital mortality in the successful and failed treatment groups, respectively.

\section{Cardiopulmonary exercise testing}

At the time of the study, nearly all patients for whom esophagectomy was planned underwent a physical endurance exercise test as a part of their routine preoperative assessment. This test is performed with the patient on a stationary ergometer; after an initial period of unloaded pedaling, a continuous, incremental increase in resistance is applied. The patient's blood pressure, pulse oximetry reading, and electrocardiogram are monitored during the test period, and the end of the test is determined by the patient's physical endurance. Typical reasons for stopping the test include leg pain, shortness of breath, and in some cases chest pain, which indicates ischemic heart disease. The results of the test are given as maximal performance during exercise in watts. Physical performance was interpreted by the department of clinical physiology, and results were divided into six categories based on age-, weight-, and sex-matched control variables: good, ordinary, slightly decreased, moderately decreased, very decreased, and extremely decreased. Data were then made dichotomous by assigning a score of 1 for a performance below good and a score of 


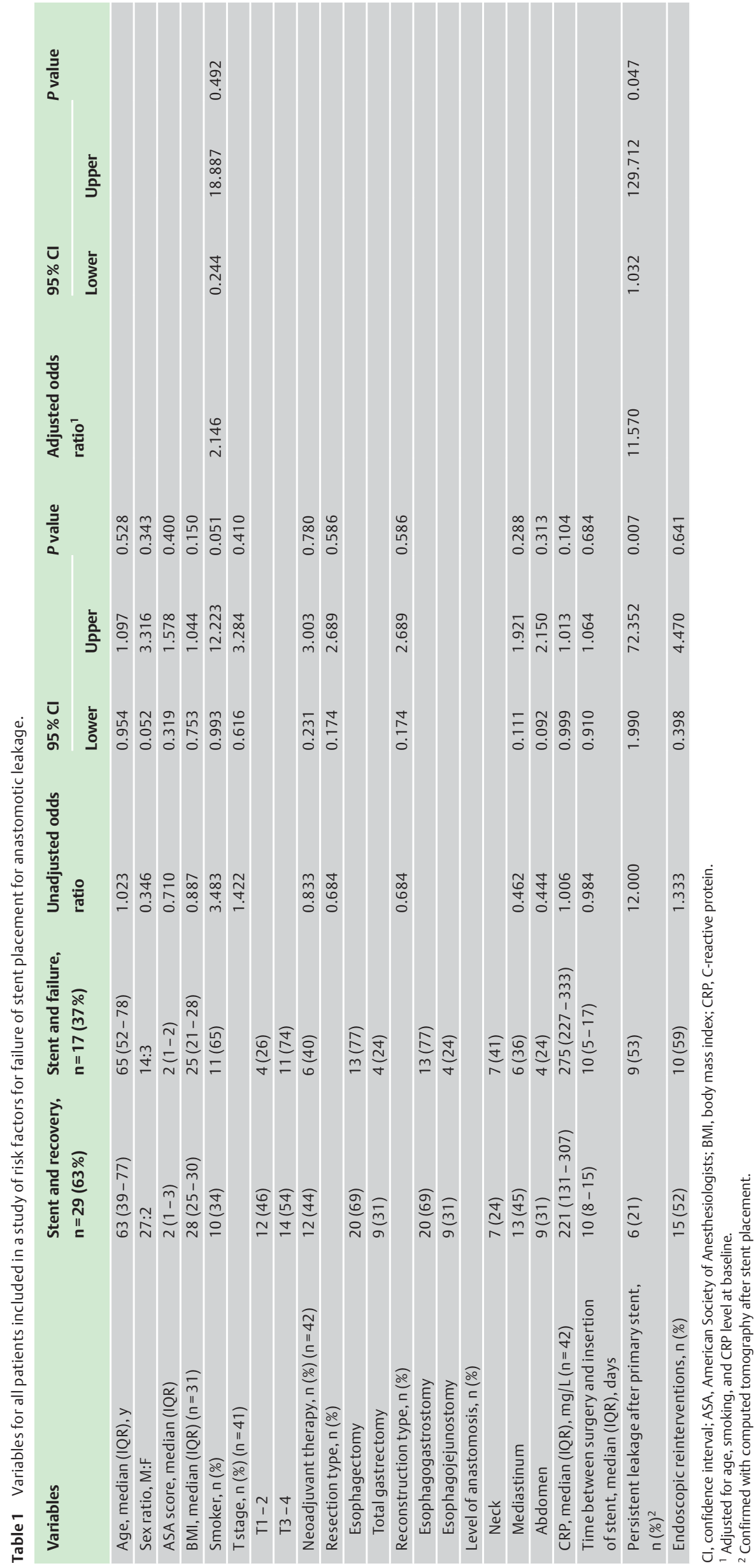




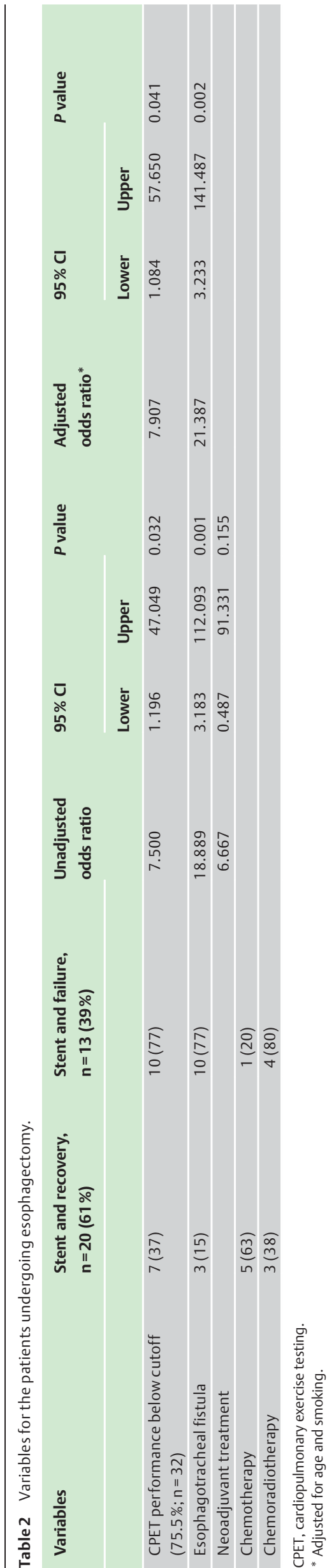

0 for a performance above good and were analyzed by receiver operating characteristics, which yielded a cutoff value of $75.5 \%$.

\section{Statistics and ethics}

Numeric data were presented as median and interquartile range (IQR). For the estimation of risk for failed SEMS therapy and outcome data, variables were analyzed with simple logistic regression. Variables with a $P$ value below 0.10 in simple logistic regression, as well as patient age and CRP level, were further analyzed with multivariable logistic regression, which was conducted by using a logistic regression model with odds ratios and 95\% confidence intervals presented as a measure of effect size. Patients who had undergone an esophagectomy were also analyzed as a separate group because some variables were applicable only to this group.Variables regarding overall treatment outcome were analyzed with Fisher's exact test, a chi-squared test, or a MannWhitney $U$ test. This study was approved by the Stockholm Ethics Committee (2013/2266 - 31/4).

\section{Results \\ $\nabla$}

\section{Preoperative characteristics of the patients}

Between January 2003 and April 2014, 266 esophagogastric anastomoses and 181 esophagojejunal anastomoses were performed. Of these, 97 were cervical anastomoses, 189 were intrathoracic, and 161 were abdominal. Anastomotic leakage occurred in 80 patients (18\%): 33 with cervical, 28 with thoracic, and 19 with abdominal anastomoses. The overall frequency of leakage was $22 \%$ for esophagogastric anastomoses and $12 \%$ for esophagojejunal anastomoses. A total of 46 patients (58\%), 41 men and 5 women, received stents as primary treatment for anastomotic leakage. The vast majority, 43 patients, had undergone surgery because of malignancy. Neoadjuvant treatment was given to 18 patients ( $42 \%$ of all those with cancer). Esophagotracheal fistula developed in 13 of the 266 patients who underwent esophagectomy $(5 \%)$.

\section{Stent treatment}

In total, 29 of the 46 patients (63\%) who received a temporary SEMS recovered successfully and were able to leave the hospital with a patent anastomosis. The overall in-hospital mortality rate in this group was $0 \%$ if only leakage-related mortality is considered. However, 1 patient in the success group spent 111 days in the hospital, and the leakage was sealed after 56 days. This patient had a previous history of both pulmonary and cardiovascular disease, and the cause of death was respiratory and cardiac failure that was not associated with the leakage or the ensuing mediastinal infection. In 17 patients (37\%), SEMS therapy failed (๑ Table1, $\odot$ Table 2). No statistically significant differences between the success and failure groups were noted regarding age, sex, comorbidities or tumor characteristics, type of resection, method of reconstruction, anatomical level of the anastomosis, or need for endoscopic reintervention after primary stenting. The median times between surgery and insertion of a SEMS, as well as the times between the diagnosis of leakage and stent insertion, were similar in the success and the failure groups. In the success group, the leak healed after a median (IQR) of $34(17-50)$ days ( $\bullet$ Table 3 ). In the failure group, 11 patients (65\%) died as a direct consequence of leakage and uncontrolled sepsis. Accordingly, the overall mortality rate in the 46 patients in whom SEMS was applied as first-line treatment was $26 \%$. 


\begin{tabular}{|llll|}
\hline Variables & $\begin{array}{l}\text { Stent and recovery, } \\
\mathbf{n = 2 9 ( 6 3 \% )}\end{array}$ & $\begin{array}{l}\text { Stent and failure, } \\
\mathbf{n = 1 7 ( 1 7 \% )}\end{array}$ & P value \\
\hline Time to recovery, median (IQR), days & $34(17-50)$ & - & \\
\hline $\begin{array}{l}\text { Time to change of treatment strategy, median } \\
\text { (IQR), days }\end{array}$ & - & $99(10-513)$ & \\
\hline Endoscopic reintervention(s), $n(\%)$ & $15(52)$ & $10(59)$ & 0.641 \\
\hline Drainage via laparotomy, $n(\%)(n=45)$ & $8(28)$ & $4(25)$ & 0.762 \\
\hline Drainage via thoracotomy, $n(\%)(n=45)$ & $2(7)$ & $4(25)$ & 0.174 \\
\hline Days in hospital, median (IQR) & $54(31-71)$ & $61(40-107)$ & 0.232 \\
\hline Days in intensive care unit, median (IQR) & $12(1-23)$ & $23(13-38)$ & 0.010 \\
\hline Ventilator support, $n$ (\%) & $19(66)$ & $16(94)$ & 0.036 \\
\hline Dialysis, $n$ (\%) & $2(7)$ & $5(29)$ & 0.083 \\
\hline In-hospital mortality, $n$ (\%) & $1(3)$ & $11(65)$ & $<0.001$ \\
\hline Second-line treatment, $n$ & & & \\
\hline Acute rescue esophagectomy & & 5 & \\
\hline Delayed colonic interposition & & 3 & \\
\hline Endoluminal vacuum therapy & & 1 & \\
\hline Esophagojejunal anastomosis & & 1 & \\
\hline
\end{tabular}

Table 3 Postoperative data for all patients.

IQR, interquartile range.

In the patients in the failure group, in whom the SEMS strategy was abandoned, the second-line treatment was initiated after a median (IQR) of 99 (10-513) days ( Table 3). Second-line treatment consisted of reoperation with elective colonic interposition in 3 cases, rescue esophagectomy with end-esophagostomy in 5 cases, and endoluminal vacuum therapy in 1 case. In 1 patient, a metastatic cancer was discovered during the in-hospital stay, and a decision was made to discontinue ICU treatment. Another patient, in whom an esophagotracheal fistula developed, underwent reoperation on 2 separate occasions. First, an attempt was made to reconstruct the esophagogastric anastomosis and suture the fistula. Because of recurrence of the fistula, reconstruction with an esophagojejunal anastomosis was subsequently performed by using a free transplanted jejunal graft. In 7 of the patients who died, a major change in treatment strategy was not considered possible because of their extremely poor clinical status, and 4 patients died despite second-line treatment.

\section{Risk factors for stent treatment failure}

Failure of the SEMS strategy was related to persistent leakage after the index stent insertion in simple logistic regression analysis $(P=0.007)$. For patients who had undergone esophagectomy, the development of a esophagotracheal fistula postoperatively and a reduced physical exercise capacity preoperatively were both statistically significant risk factors for SEMS failure $(P=$ 0.001 and $P=0.032$, respectively). In the multivariable logistic regression analyses, adjustments were made for CRP level at baseline, smoking status, and age, after which persistent leakage after initial SEMS remained a statistically significant risk factor for failure $(P=0.047)$. In the corresponding analysis for patients with an esophagectomy, both reduced physical exercise capacity and esophagotracheal fistula remained statistically significant risk factors ( $P=0.041$ and $P=0.002$, respectively) after adjustment for age and smoking status. However, it was not possible to adjust for sex in any of the analyses because of a skewed distribution with only 2 women in the success group (7\%) and 3 in the failure group (18\%).
Treatments other than placement of a self-expandable metal stent

A total of 34 patients (43\%) did not receive a SEMS as primary treatment. The reasons for applying alternative treatments were as follows: 13 patients had a cervical anastomosis that was primarily treated with débridement of the cervical wound because the esophagogastric anastomosis was considered too proximally located to allow a SEMS to be successfully deployed, and 9 patients had subclinical leakage that was assessed as best managed by conservative means. In 4 patients who presented with extensive leakage, with or without dramatic clinical deterioration, SEMS was not considered justified and immediate surgical intervention was mandated. Another 8 patients demonstrated signs of conduit necrosis at the emergency endoscopy in the near vicinity of or including the anastomosis and required rescue surgery. The overall in-hospital mortality rate of those patients in whom SEMS placement was not suitable was $18 \%$.

\section{Discussion}

$\nabla$

During the studied decade, we consistently and prospectively applied an esophageal SEMS-based strategy as a part of the multimodal first-line treatment of dehiscence of either an esophagogastric or esophagojejunal anastomosis. Therefore, the current study for the first time provides a comprehensive analysis of how often such a therapeutic strategy can be used and its results. The focus of the study was on risk factors for the failure of this therapeutic concept, which we defined as a radical change of treatment strategy because of uncontrolled mediastinitis, which in this setting meant emergency esophagectomy with end-esophagostomy, or as death resulting from leakage and uncontrolled sepsis. We have shown that in patients in whom the leakage was controlled with the SEMS concept, the in-hospital mortality rate was low. The majority of patients who did not receive a SEMS had cervical anastomoses, which could be carefully managed by externalization and drainage through a wide opening of the neck incision, allowing direct cleansing. In total, $43 \%$ of the patients did not receive a SEMS as primary therapy, of whom $12 \%$ required an emergency operation because of severe mediastinitis. The 
other main circumstance in which SEMS treatment was inappropriate was conduit necrosis.

In $58 \%$ of the cases, SEMS was applied as first-line therapy with the objective of sealing the leak. We were able to demonstrate several risk factors for failure of this treatment strategy in patients with esophagogastric or esophagojejunal anastomotic leakage. Persistent leakage after the first stent placement was identified as an independent risk factor for failure in the whole cohort, which we believe is of clinical significance. This strongly suggests that endoscopic reintervention with adjustment or change of stent should always be considered in a patient in whom signs of continuous leakage develop if there is proof of a sealed leak after the first stent insertion. We therefore suggest that radiologic monitoring after stent placement be done routinely because such information is crucial for important decisions that need to be taken later during the course of treatment.

In the esophagectomy group, additional risk factors, not applicable to the gastrectomy group, were analyzed. The formation of an esophagotracheal fistula emerged as an independent risk factor for the failure of stent-based treatment. The currently reported incidence of airway fistulization is $5 \%$ for all esophagogastric anastomoses, which is within the range reported in the few larger series on this devastating complication $[21,23]$. Our experience of treating this specific complication with SEMS, usually applying a dual-SEMS strategy, has been presented before [22]. When an esophagotracheal fistula develops after placement of a stent over an anastomotic leak, it cannot be ruled out that the airway fistula has been caused by pressure from the esophageal stent on the very frail pars membranacea of the trachea. Esophagotracheal fistulas also develop in the absence of stents, and with the currently available data, it is not possible to identify cases in which there is a risk for inducing the formation of an esophagotracheal fistula with a SEMS. In the multivariable logistic regression analyses, adjustments were made for CRP level at baseline, smoking status, and age, and reduced physical working capacity, as assessed during the preoperative work-up, remained a statistically significant risk factor after these adjustments. However, it was not possible in any of the analyses to adjust for sex because of a skewed distribution with only 2 women in the success group ( 7 $\%$ ) and 3 in the failure group (18\%).

Significant clinical experience and research suggest that patients with a poor physical performance status preoperatively have an increased risk for mortality after esophagectomy [24-26], which is related to a generally poor outcome after surgery and susceptibility to complications. This was the main reason why we analyzed the possible influence of reduced physical performance on the risk for failure after SEMS treatment of an already established postoperative complication. In fact, this emerged as an independent risk factor, which may improve the risk stratification of patients eligible for esophagectomy. Given the observations presented herein, it may be suggested that rescue esophagectomy be considered at an early time in patients with a reduced physical performance when anastomotic leakage develops that does not promptly respond to SEMS treatment.

This study has some weaknesses that need to be carefully considered. First of all, despite the fact that we tried to follow a predefined management strategy, the study was retrospective, and as such, some vital information may have avoided detection. Moreover, in $43 \%$ of the patients, stents were not applied as a primary treatment. On the other hand, and as presented previously, there were relevant reasons for not doing so. The lower mortality rate among the patients who did not receive a stent implies that their cases were less complicated. Lastly, the patient cohort is by necessity heterogeneous, given that it includes both patients with esophagogastric and patients with esophagojejunal anastomoses. Because of the limited number of patients, further subgroup studies would have introduced a significant risk for loss of power, which is why we completed a similar analysis of risk factors in those who underwent esophagectomy alone.

Whenever a SEMS-based strategy is followed in a clinical situation as demanding as the one currently presented, the team has to be prepared to undertake repeated endoscopic reinterventions to control ongoing or recurrent leakage. In this context, it should be emphasized that the frequency of reintervention did not emerge as a statistically significant risk factor when the success and failure groups were compared. However, the conclusions that can be drawn from the present study are that SEMS can be applied up front in patients with esophagogastric or esophagojejunal anastomotic leakage as part of a carefully processed treatment strategy. Treatment failure must be vigorously searched for, and the main risk factors for failure are continued leakage from the anastomosis and the development of an airway fistula. If these events occur in a patient with a reduced physical capacity preoperatively, aggressive therapeutic alternatives have to be promptly instituted. Currently, it appears that emergency esophagectomy may have been delayed in some of our patients because the second-line treatment was initiated after a median (IQR) of 99 (10-513) days. Future clinical research must better document the options that can add to the efficacy of SEMS (e.g., vacuum therapy) and optimize timing of the switch to secondline treatment.

\section{Competing interests: None}

\section{References}

1 Sauvanet A, Mariette C, Thomas $P$ et al. Mortality and morbidity after resection for adenocarcinoma of the gastroesophageal junction: predictive factors. J Am Coll Surg 2005; 201: 253-262

2 Zilling T, Olseen P, Walther BS. Prediction of hospital stay after total gastrectomy. Anticancer Res 1997; 17: 1355-1359

3 Lang H, Piso P, Stukenborg C et al. Management and results of proximal anastomotic leaks in a series of 1114 total gastrectomies for gastric carcinoma. Eur J Surg Oncol 2000; 26: 168-171

4 Sierzega M, Kolodziejczyk P, Kulig J et al. Impact of anastomotic leakage on long-term survival after total gastrectomy for carcinoma of the stomach. Br J Surg 2010; 97: 1035-1042

5 Meyer L, Meyer F, Dralle $H$ et al. Insufficiency risk of esophagojejunal anastomosis after total abdominal gastrectomy for gastric carcinoma. Langenbecks Arch Surg 2005; 390: 510-516

6 Djarv T, Derogar M, Lagergren P. Influence of co-morbidity on longterm quality of life after oesophagectomy for cancer. Br J Surg 2014; 101: $495-501$

7 Sarela AI, Tolan DJ, Harris $K$ et al. Anastomotic leakage after esophagectomy for cancer: a mortality-free experience. J Am Coll Surg 2008; 206: $516-523$

8 Turkyilmaz A, Eroglu A, Aydin Yet al. The management of esophagogastric anastomotic leak after esophagectomy for esophageal carcinoma. Dis Esophagus 2009; 22: 119-126

9 Urschel JD. Esophagogastrostomy anastomotic leaks complicating esophagectomy: a review. Am J Surg 1995; 169: 634-640

10 Whooley BP, Law S, Alexandrou A et al. Critical appraisal of the significance of intrathoracic anastomotic leakage after esophagectomy for cancer. Am J Surg 2001; 181: 198 - 203

11 Migita K, Takayama T, Matsumoto S et al. Risk factors for esophagojejunal anastomotic leakage after elective gastrectomy for gastric cancer. J Gastrointest Surg 2012; 16: 1659-1665

12 Schardey HM, Joosten $U$, Finke $U$ et al. The prevention of anastomotic leakage after total gastrectomy with local decontamination. A prospective, randomized, double-blind, placebo-controlled multicenter trial. Ann Surg 1997; 225: 172-180 
13 Alanezi K, Urschel JD. Mortality secondary to esophageal anastomotic leak. Ann Thorac Cardiovasc Surg 2004; 10: 71 - 75

14 Brangewitz M, Voigtlander T, Helfritz FA et al. Endoscopic closure of esophageal intrathoracic leaks: stent versus endoscopic vacuum-assisted closure, a retrospective analysis. Endoscopy 2013; 45: 433-438

15 Rodella L, Laterza E, De Manzoni $G$ et al. Endoscopic clipping of anastomotic leakages in esophagogastric surgery. Endoscopy 1998; 30: 453 456

16 Bohm G, Mossdorf A, Klink C et al. Treatment algorithm for postoperative upper gastrointestinal fistulas and leaks using combined vicryl plug and fibrin glue. Endoscopy 2010; 42: 599-602

17 Choi HJ, Lee BI, Kim JJ et al. The temporary placement of covered selfexpandable metal stents to seal various gastrointestinal leaks after surgery. Gut Liver 2013; 7: 112-115

18 Persson S, Elbe P, Rouvelas I et al. Predictors for failure of stent treatment for benign esophageal perforations - a single center 10-year experience. World J Gastroenterol 2014; 20: 10613-10619

19 Hoeppner J, Kulemann B, Seifert $G$ et al. Covered self-expanding stent treatment for anastomotic leakage: outcomes in esophagogastric and esophagojejunal anastomoses. Surg Endosc 2014; 28: 1703-1711
20 Feith M, Gillen S, Schuster T et al. Healing occurs in most patients that receive endoscopic stents for anastomotic leakage; dislocation remains a problem. Clin Gastroenterol Hepatol 2011; 9: 202 - 210

21 Schweigert M, Solymosi N, Dubecz A et al. Endoscopic stent insertion for anastomotic leakage following oesophagectomy. Ann R Coll Surg Engl 2013; 95: 43-47

22 Elbe $P$, Lindblad $M$, Tsai $J$ et al. Non-malignant respiratory tract fistula from the oesophagus. A lethal condition for which novel therapeutic options are emerging. Interact Cardiovasc Thorac 2013; 16: 257-262

23 Al-issa MA, Petersen TI, Taha AY et al. The role of esophageal stent placement in the management of postesophagectomy anastomotic leak. Saudi J Gastroenterol 2014; 20: 39-42

24 Feeney C, Hussey J, Carey $M$ et al. Assessment of physical fitness for esophageal surgery, and targeting interventions to optimize outcomes. Dis Esophagus 2010; 23: 529-539

25 McCulloch P, Ward J, Tekkis PP. Mortality and morbidity in gastro-oesophageal cancer surgery: initial results of ASCOT multicentre prospective cohort study. BMJ 2003; 327: $1192-1197$

26 Ferguson MK, Durkin AE. Preoperative prediction of the risk of pulmonary complications after esophagectomy for cancer. J Thorac Cardiovasc Surg 2002; 123: $661-669$ 